Somnologie $2018 \cdot 22: 240-244$ https://doi.org/10.1007/s11818-018-0185-1 Eingegangen: 10. September 2018 Angenommen: 19. Oktober 2018 Online publiziert: 5. November 2018 (c) Der/die Autor(en) 2018

CrossMark

\author{
Nikolaus Netzer ${ }^{1,2,3}$ (D) Stephan Pramsohler ${ }^{1} \cdot$ Helmut Frohnhofen ${ }^{4}$ \\ 'Hermann Buhl Institut für Hypoxie und Schlafmedizinforschung, Institut für Sportwissenschaften, \\ Fakultät für Psychologie und Sportwissenschaften, Leopold Franzens Universität Innsbruck, Bad Aibling \\ Deutschland \\ ${ }^{2}$ Institute for Mountain Emergency Medicine, Eurac Research, Bozen, Italien \\ ${ }^{3}$ Abteilung Sportmedizin und Rehabilitation, Medizinische Klinik II, Medizinische Universitätsklinik Ulm, \\ Ulm, Deutschland \\ ${ }^{4}$ Abteilung Geriatrie, Krupp Krankenhaus Essen, Universität Witten Herdecke, Essen, Deutschland
}

\section{Schlaf beim alten Menschen}

\section{Einleitung}

Die demografische Entwicklung in den meisten Industrieländern führt dazu, dass der durchschnittliche Patient in Klinik und Praxis generell älter ist als noch vor der Jahrtausendwende. In Deutschland rechnen die Statistiker damit, dass mehr als $50 \%$ der Gesamtbevölkerung 2030 über 60 Jahre alt sein werden.

Dementsprechend wird die Mehrheit der Patienten im Schlaflabor in Kürze Senioren sein bzw. ist es je nach Schlaflabortyp (ambulant, stationär, Schwerpunkt neurologisch oder pulmologisch, regionaler Standort) jetzt schon. Die angloamerikanische Literatur definiert älter, geriatrisch, ab dem Rentenalter von 65 Jahren, die gesetzlichen Krankenkassen in Deutschland ab dem 70. Lebensjahr.

Wichtig für alle medizinischen Berufe ist daher zunächst ein Verständnis des normalen Schlafes des älteren Menschen, denn was normal ist und was pathologisch bzw. therapiebedürftig, wird erst durch die für viele Menschen verlängerte Lebensspanne zwischen Renteneintrittsalter und Tod so richtig bedeutsam. In dieser Lebensspanne bekommt das subjektive Alter eines Menschen Einfluss darauf wie der Einzelne seinen Schlaf empfindet [1]. Ob eine 70-jährige Frau z. B. $75 \%$ Schlafeffizienz als altersbedingt normal empfindet oder sich von ihrer Hausärztin wegen Insomnie ein Schlafmittel verschreiben lässt, hängt von diesem subjektiven Alter ab. Es fehlt derzeit in der Medizin durch mangelndes Wissen über normalen Schlaf noch das professionelle
Korrektiv, was leider zu oft zu unnötigen medikamentösen Therapieversuchen führt. Im höheren Lebensalter stehen gesellschaftlich normatives Schlafverhalten (7-8h nächtliches Durchschlafen) und altersbedingt natürliches Schlafverhalten (verlängerte Einschlafzeit, frakturierter Schlaf) mehr im Widerspruch als in der Ausbildungs- und Berufszeit [2].

Für die Unterscheidung zwischen normalem und pathologischem Schlafbei älteren Menschen ist zunächst die Unterscheidung zwischen normalem, gesunden Altern und im Alter häufiger auftretenden Erkrankungen wie z. B. Demenz, Herzinsuffizienz, Gelenkserkrankungen u. a. notwendig. Schlafstörungen bei Senioren können oft sekundärer Natur sein (z.B. komplexe Schlafapnoe bei Herzinsuffizienz, Hypersomnie und gestörter circadianer Rhythmus bei Demenz) [3]. Eine gute schlafmedizinische Diagnostik setzt also die Kenntnis über bestehende Grunderkrankungen, besonders auch beim älteren Patienten voraus.

Schlaf beim älteren Menschen ist ein zunehmend interessantes Forschungsthema geworden und durch Metaanalysen von Schlafstudien und altersbezogener Analysen großer klinischer Studien wie der „Sleep, Heart and Health“ können wir heute mehr über den normalen und pathologischen Schlaf bei älteren Menschen erfahren als noch zu Beginn des Jahrhunderts [4-6].

\section{Schlafarchitektur beim älteren Menschen}

Bei der Betrachtung veränderter Schlafarchitektur im höheren Lebensalter muss grundsätzlich bedacht werden, dass unterschiedliche Auswertekriterien (Rechtschaffen und Kales oder American Academy of Sleep Medicine [AASM] 2007-2017) bereits zu Fehlbeurteilungen aus älteren Untersuchungen führen können. Dies gilt auch für Analysen aus sehr lang andauernden Studien, wie der „Sleep Heart Health“, während derer Dauer sich die Auswertekriterien der AASM geändert haben.

Für die Beurteilung des Elektroenzephalogramms (EEG) beim älteren Menschen ist entscheidend, dass die Amplituden aller Wellen im Alpha- bis Deltabereich mit dem Alter abnehmen. Bei den normalerweise hochamplitudigen Deltawellen fällt dies besonders auf, sie fallen z.T. beim gesunden älteren Menschen deutlich unter $75 \mathrm{uV}$. Die zeitliche Dauer der Entladungen verändert sich kaum. Die Frequenzen $(1-12 \mathrm{~Hz})$ in den Schlafstadien bleiben also gleich [7]. Der Grund für dieses Phänomen dürfte beim gesunden älteren Menschen die physiologisch bedingte Abnahme von Synapsen und vor allem myelinummantelter Nervenfasern sein [8]. Dies hat nichts mit dem Untergang von Nervenzellen bei Demenz zu tun. Delta- bzw. Tiefschlaf wird deshalb im Routineschlaflabor bzw. von automatischen Auswertesystemen oft unterschätzt und fälschlicherweise als Non-REM 2 ausgegeben. Allerdings zeigen sich die Rückgänge der 
Tab. 1 Schlafstadienverteilung (Durchschnitt ohne Standardabweichung in Prozent der totalen Schlafzeit) bei 2685 weitgehend gesunden Personen, ohne diagnostizierbare Schlafstörungen in der Polysomnographie (PSG) und ohne Schmerzsymptomatik, in den verschiedenen Altersgruppen aus der Sleep Heart Health Studie [4]

\begin{tabular}{|c|c|c|c|c|c|c|c|c|}
\hline \multirow[b]{2}{*}{$\begin{array}{l}\text { Alter } \\
\text { (Jahre) }\end{array}$} & \multicolumn{2}{|c|}{ Non-REM 1} & \multicolumn{2}{|c|}{ Non-REM 2} & \multicolumn{2}{|c|}{$\begin{array}{l}\text { Delta } \\
\text { (Tief)-Schlaf }\end{array}$} & \multicolumn{2}{|c|}{ REM-Schlaf } \\
\hline & Männer & Frauen & Männer & Frauen & Männer & Frauen & Männer & Frauen \\
\hline $37-54$ & 5,8 & 4,6 & 61,4 & 58,5 & 11,2 & 14,2 & 19,5 & 20,9 \\
\hline $55-60$ & 6,3 & 5,0 & 64,5 & 56,2 & 8,2 & 17,0 & 19,1 & 20,2 \\
\hline $61-70$ & 7,1 & 5,0 & 65,2 & 57,3 & 6,7 & 16,7 & 18,4 & 19,3 \\
\hline$>70$ & 7,6 & 4,9 & 66,5 & 57,1 & 5,5 & 17,2 & 17,8 & 18,8 \\
\hline
\end{tabular}

\section{Tab. 2 Praktische Aspekte bei älteren Menschen in der Polysomnographie (๑ N. Netzer}

Die Polysomnographie und Polygraphie hochbetagter Menschen unterscheidet sich in einigen Punkten von denen bei jüngeren und mittelalten Patienten. Hier eine Zusammenstellung der zehn wichtigsten Erfahrungswerte aus fünfzehn Jahren geriatrischem Schlaflabor:

1. Unbeobachtete Polygraphien und Polysomnographien lassen sich oft bei älteren Menschen schlecht realisieren, daher sollte die Polygraphie zu Hause oder im Pflegeheim unter Beobachtung des Pflegepersonals bzw. der Angehörigen stattfinden. Letztere sollten im Falle einer Polygraphie beim Anlegen des Polygraphen oder Typ 4-Geräts helfen.

2. Für ambulante Polysomnographien in der niedergelassenen Schlafmedizinpraxis ohne zusätzlich zum Schlaflabor-Assistenten anwesendes Pflegepersonal sind hochbetagte Patienten wegen des erhöhten pflegerischen Aufwandes selten geeignet.

3. Die Polysomnographie sollte in einer Umgebung stattfinden, die dem Patienten bereits vertraut ist. Daher haben wir in unserer Klinik die Polysomnographen auf Untersuchungswägen montiert und untersuchen die Patienten frühestens drei bis vier Tage nach der stationären Aufnahme im eigenen Patientenbett.

4. Bettpfanne und Urinflasche sollten in Reichweite sein, ebenso Notfallmedikamente u.a.

5. Es muss damit gerechnet werden, dass der plötzlich aufwachende, auch der vorher kognitiv nicht auffällig gewordene ältere Patient verwirtt ist und sich durch die Untersuchungssituation bedroht fühlt.

6. Wegen des erhöhten Hautwiderstandes bei älteren Patienten mit trockener Haut muss die Elektrodenpaste bzw. das Gel von Einmalelektroden erst einziehen, bevor die Signale vernünftig erscheinen.

7. Wegen der geringeren Amplituden muss mit teilweise schwächerem EEG-Signal gerechnet werden. Gleiches gilt für die REM-Intensität (Augenbewegungen). Daher kommt es bei automatisierter Auswertung und beim nicht geriatrisch erfahrenen Auswerter öfters zu Fehlern beim Schlafstadienscoring. Delta- und REM-Schlaf werden zu niedrig ausgewertet und daher fälschlicherweise eine Schlafstörung diagnostiziert.

8. Da die Grundsauerstoffsättigung bereits etwas niedriger liegt und Entsättigungen bei respiratorischen Ereignissen oft nicht so ausgeprägt sind, kommt es bei automatisierter Auswertung zu falschen ODIs

9. Da viele ältere Menschen $\beta$-Blocker oder andere Kardiaka einnehmen, sind Herzfrequenzanstiege nach respiratorischen und nicht respiratorischen Arousals nicht so ausgeprägt, Arousals müssen daher in erster Linie aus dem EEG abgeleitet werden.

10. In der nächtlichen Routinepolysomnographie fallen Naps, die vorher tagsüber stattgefunden haben, unter den Tisch. Die Gesamtschlafzeit wird daher zu niedrig bewertet. Der ältere Mensch sollte vor der Polysomnographie über Schlafzeiten davor tagsüber befragt und diese Zeit zur TST aus der Polysomnographie hinzuaddiert werden.
Amplituden im Deltabereich wesentlich deutlicher bei Männern als bei Frauen.

Geht man von einer altersberücksichtigenden, korrekten, manuellen Auswertung aus, dann ergeben sich Hinweise auf einen deutlichen Rückgang an Tiefschlaf bei Männern über 70 Jahren auf 4,5-6,5\% ,total sleep time“ (TST) gegenüber mittelalten Männern (9,9-12,6\% TST) und bei Frauen kein Rückgang, eher ein Anstieg vom im Schnitt 14,2\%TST auf $17,2 \%$ TST [4]. Dementsprechend steigen bei Männern die Non-REM („rapid eye movement“)-Stadien 1 und 2 an und bei Frauen reduzieren sie sich leicht. Diese unterschiedliche Stadienverteilung der Geschlechter ist wegen der oben beschriebenen Schwierigkeit, Delta-Schlaf vor allem bei älteren Männern genau von Non-REM 2 abzugrenzen, umstritten [7].

Bei beiden Geschlechtern gibt es einen minimalen Rückgang des prozentualen REM-Anteils. Die Augenbewegungsdichte nimmt im Alter ab und macht es sowohl für manuelle Auswerter als auch für die automatisierte Auswertung schwieriger, REM-Schlaf leicht zu identifizieren [8]. Übereinstimmend haben Meta- und Langzeitanalysen ergeben, dass vom mittleren Alter zu den über 70-Jährigen die Schlafeffizienz leicht abnimmt von 85 auf $80 \%$ im Schnitt und dies unabhängig vom Geschlecht. Beim gesunden Alterungsprozess liegt die Schlafeffizienz damit aber immer noch in einem Bereich für erholsamen Schlaf.

Die Häufigkeit der nicht respiratorischen und nicht beinbewegungsbezogenen Arousals nimmt im Alter zu, sie steigt auf 18-27 Arousals pro Stunde an [9] Gesunde ältere Menschen wachen auch öfters während des Schlafes auf als jüngere Menschen, haben aber in der Regel kein Problem wieder einzuschlafen ([10]; - Tab. 1).

\section{Circadiane Rhythmik beim älteren Menschen}

Es gilt grundsätzlich, dass die circadiane Rhythmik beim gesunden älteren Menschen weniger stark ausgeprägt ist als beim jüngeren und mittelalten Menschen. Der Grund dafür dürfte in der 
reduzierten Produktion bzw. Ausschüttung von circadian wirksamen Hormonen und Neurotransmittern (Melatonin, Cortisol, Östrogen, Dopamin, Orexin u. a.) liegen. Eine reduzierte Melatoninfreisetzung bei beginnender Dunkelheit verstärkt noch bei Senioren mit grauem Star, würde auch Phasenverschiebungen erklären, insbesondere hin zu einer verspäteten Einschlafphase („delayed phase syndrome“). Dass ältere Menschen diesen physiologischen Vorgang als unangenehm und nicht gesellschaftlich normativ empfinden und daher ihre Bettgehzeiten immer weiter nach vorne verlegen, um dies zu kompensieren, hat in der Vergangenheit oft dazu geführt, dass Schlafforscher dies als verfrühte Einschlafphase („advanced phase syndrome“) missinterpretiert haben [11]. Bei Desynchronisierungs (Bunker)-Studien hat sich gezeigt, dass ältere gesunde Menschen grundsätzlich mehr Aufwachphasen während der Schlafperioden haben als jüngere. Dies hatte jedoch keinen Einfluss auf die Gesamtschlafzeit. Generell gilt, dass eine reduzierte Gesamtschlafzeit deutlich unter $7 \mathrm{~h}$ bei älteren Menschen als Hinweis auf Erkrankungen im Alter gelten muss und selbst, wie auch bei jüngeren Menschen, als Risikofaktor für diverse Folgeerkrankungen bzw. als erhöhtes Mortalitätsrisiko gilt [2].

Eine Entwicklung vom monophasischen Nachtschlaf hin zum polyphasischen Schlaf über den Tag verteilt ist möglicherweise beim älteren Menschen nicht nur eine Folge von Veränderungen im Arbeits- und Sozialleben, sondern wie Resultate aus der Tierforschung von Säugetieren bis hin zu Drosophila zeigen ein natürlicher Altersprozess. Auch für Tiere gilt jedoch, dass die Gesamtschlafzeit im Alter nicht abnimmt [12]. Im Gegenteil dürfte die Gesamtschlafzeit sowohl bei Tieren wie auch beim Menschen im Alter etwas zunehmen [13]. Eine Gesamtschlafzeit von bis zu $9 \mathrm{~h}$ am Tag kann beim älteren Menschen normal sein. Dies ist insofern wichtig als bei der Beurteilung positiver und negativer Effekte von Naps bzw. deutsch Nickerchen am Tage tatsächlich entscheidend ist welche Gesamtschlafzeit sich ergibt. Resultiert aus der Gesamtschlafzeit der Hinweis auf eine Hypersomnie (TST am

Somnologie 2018 $22: 240-244$ https://doi.org/10.1007/s11818-018-0185-1

(c) Der/die Autor(en) 2018

\section{N. Netzer · S. Pramsohler · H. Frohnhofen}

\section{Schlaf beim alten Menschen}

\section{Zusammenfassung}

Die demografische Entwicklung führt zu einer Zunahme älterer Patienten in der Schlafmedizin. In den Grundsätzen gelten für geriatrische Patienten die gleichen Leitlinien und Verfahrensweisen wie für jüngere und mittelalte Patienten. Es gibt jedoch physiologische und pathophysiologische Unterschiede in Bezug auf den Schlaf älterer Menschen, die Beachtung finden sollten, damit es nicht zu Fehlbehandlungen kommt und damit Ärzte aller Berufsgruppen bessere schlafmedizinische Auskünfte gegenüber Patienten und gesundheitsinteressierten Senioren geben können.

Die altersbezogenen Veränderungen des Schlafes finden, soweit bisher untersucht, bereits vor dem 70 . Lebensjahr statt, also im mittleren und Jungseniorenalter: Generell nehmen im Elektroenzephalogramm (EEG) die Potentialstärken elektrischer Impulse ab. Deltaschlaf (N3) reduziert sich beim gesunden Altern, bevorzugt bei Männern, genauso um wenige Prozentpunkte der Gesamtschlafzeit wie REM(„rapid eye movement “)-Schlaf und die Schlafeffizienz nimmt geringfügig ab. Die Gesamtschlafzeit verändert sich nicht, aber Schlaf wird frakturierter, nicht respiratorische Arousals und die Einschlafzeit nehmen etwas zu. Der $\mathrm{CO}_{2}$-gesteuerte Atemantrieb und damit auch der "loop gain" nach Apnoen nimmt leicht ab, allerdings im Gegenzug auch die Elastizität des Gewebes und der Muskulatur der oberen Atemwege, sodass obstruktive Apnoen und Hypopnoen leicht physiologisch bedingt zunehmen. Pathologische Schlafveränderungen beim älteren Menschen, bedingt z. B. durch dementielle Entwicklungen, respiratorische, kardiovaskuläre Erkrankungen und Schmerzsyndrome sind in ihrer Relation zum gesunden Altern noch unzureichend untersucht.

Schlüsselwörter

Altern · Apnoe · Arousal · EEG · REM-Schlaf

\section{Sleep in the elderly}

\section{Abstract}

Demographic development has led to an increase in elderly and geriatric patients requiring sleep medicine. In principle, the same guidelines and procedures apply to elderly patients as apply for younger patients. However, there are physiological and pathophysiological differences in relation to the sleep of the elderly that should be given attention to avoid mistreatment, so that all medical professionals are able to give patients and health-conscious seniors better information about sleep medicine. Age-related changes in sleep, according to research so far, start before the age of 70 during middle and early senior age. Generally, electrical potentials in EEG signals decrease. Slow-wave sleep is reduced in healthy old age, especially in men, as few percentage points of the total sleep time (TST) as REM sleep, and sleep efficiency decreases slightly, TST does not change. Sleep is more fractured with more nonrespiratory arousals, and the time needed to fall asleep increases somewhat. Respiratory drive steered through blood $\mathrm{CO}_{2}$, and therefore loop gain after apnea, decrease slightly with aging. However, the elasticity of the tissue and musculature of the upper airways do too; thus, obstructive apnea and hypopnea show a slight physiological increase. Pathological changes in sleep in older people caused by, for example, the development of dementia, respiratory and cardiovascular diseases, and pain syndromes have not yet been sufficiently investigated in relation to sleep changes with healthy aging.

Keywords

Aging · Apnea - Arousal - Electroencephalography $\cdot$ Sleep, REM
Tag deutlich über 9h), so kann dies ein Hinweis auf verschiedenste Erkrankungen wie Demenz oder klinisch relevante Schlafapnoe sein und mit einer erhöhten Mortalität einhergehen. Bleibt die TST am Tag im normalen Korridor von 6,5-9h, so können die Naps auch als Powernaps mit positivem Effekt auf ein gesundes Altern gesehen werden.

Inzwischen gut dokumentiert ist, dass mit zunehmendem Alter die Fähigkeit der willkürlichen (Schlaf nachholen) 


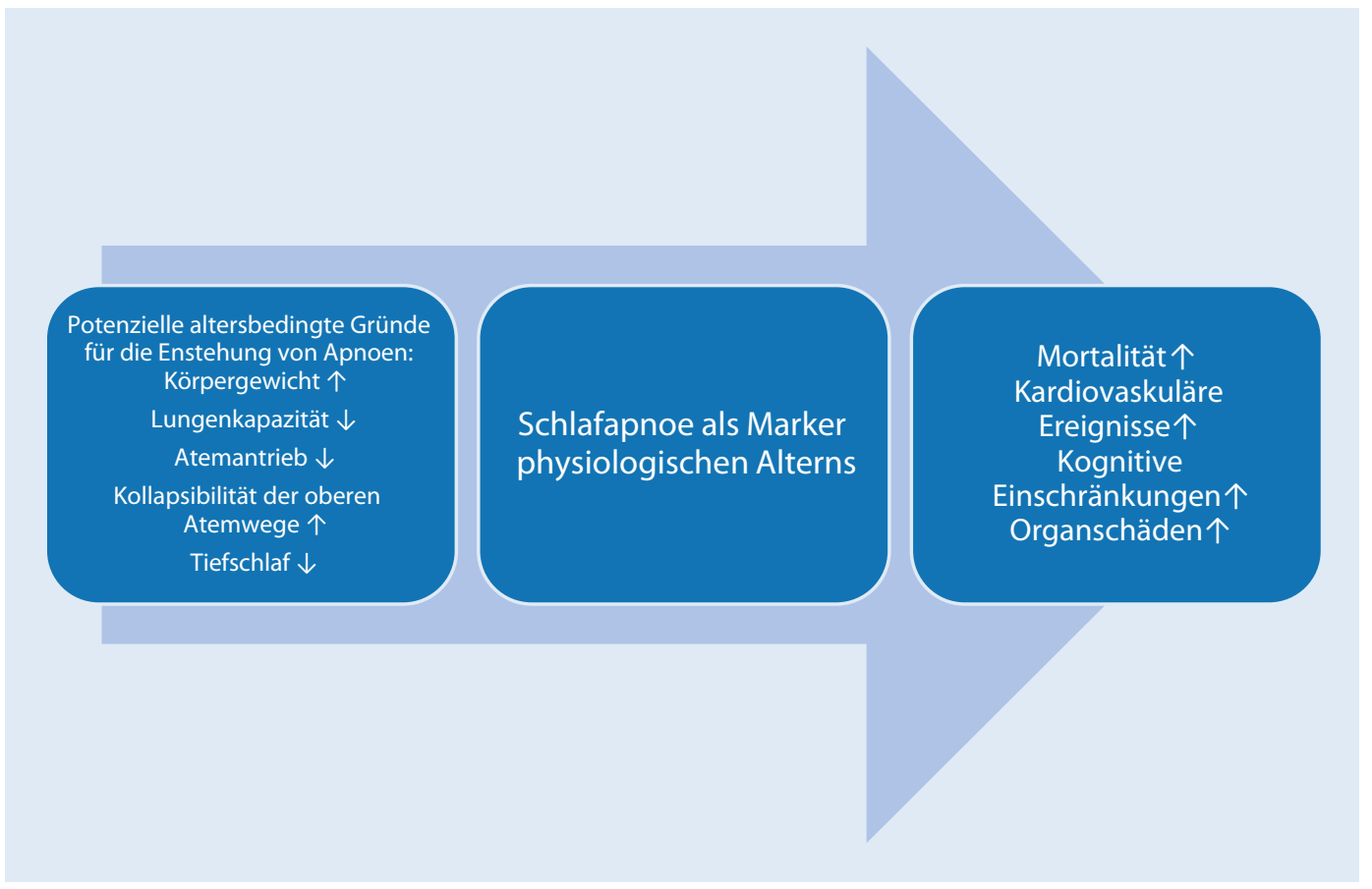

Abb. $1<$ Diskutierte Zusammenhänge zwischen erhöhter Prävalenz für Schlafapnoe im Alter und möglichen klinischen Folgen (@ N. Netzer [24]) oder Lichteinfall bedingten Phasenverschiebung (Jet lag kompensieren) kontinuierlich abnimmt. Diese reduzierte Möglichkeit der willentlich beeinflussbaren Phasenverschiebung (Schlaf nachholen) im Alter gilt auch für andere Säugetiere [14-16]. Aus diesem Grund ist Schichtarbeit für Menschen ab einem Alter von über 50 Jahren noch belastender als für jüngere Menschen. Die Rückkehr in den normalen circadianen Rhythmus nach Interkontinentalflugreisen dauert bereits ab dem 30. Lebensjahr mit jedem Lebensjahr länger. Die immer noch gültige wissenschaftliche These dafür, warum dies so ist, bezieht sich auf strukturelle Veränderungen im Nucleus suprachiasmaticus ab einem bestimmten Alter, vermutet wird bereits ab dem 20 . Lebensjahr [17]. Offensichtlich nimmt hier die Sensitivität für Melatonin linear zum Älterwerden ab. Veränderungen der circadianen Rhythmik im Alter können damit also sowohl mit geringerer $\mathrm{Me}$ latoninausschüttung als auch geringerer Melatoninsensitivität zusammenhängen.

\section{Schlaf und Atmung beim älteren Menschen}

In der Schlafmedizin besteht die Diskussion, ab wann die verschiedenen Formen von Schlafapnoe beim älteren Men- schen behandelt werden sollten, sprich ob bei älteren Menschen höhere ApnoeIndices tolerabel und als altersphysiologisch einzustufen sind. Medizinisch vernünftig ist es natürlich, jede Schlafapnoe beim älteren Menschen nach den gleichen Grundsätzen zu behandeln wie beim jüngeren und mittelalten Menschen und zwar entsprechend dem Vorhandensein klinischer Symptome wie vermehrte Tagesmüdigkeit.

Grundsätzlich gilt: Obstruktive Apnoen und Hypopnoen nehmen beim älteren Menschen aus altersphysiologisch bedingten Gründen zu und damit die Prävalenz für eine Schlafapnoe gemäß Definition nach klassischen AHI(ApnoeHypopnoe-Index)-Grenzwerten [18].

Bei beiden Geschlechtern, statistisch gesehen bevorzugt bei Männern, kommt es zu einer höheren Kollapsibilität der oberen Atemwege und einem höheren Atemwegswiderstand in den oberen Atemwegen durch den Verlust elastischer Fasern im Bindegewebe, Fetteinlagerung und einen leichten Abbau der Pharynxmuskulatur [19]. Bevorzugt bei Männern (siehe oben) trägt eine Stadienumverteilung vom apnoearmen Delta-Schlaf hin zu den Non-REM-Stadien 1 und 2 ebenfalls zu mehr obstruktiven Apnoen und Hypopnoen bei, bei Frauen verstärkt sich die Kollapsibilität der oberen Atemwege durch die Reduktion der positiv auf die Muskelspannung der oberen Atemwege wirkenden weiblichen Sexualhormone. Insbesondere der Abfall des Progesteronspiegels spielt hier die entscheidende Rolle [20, 21].

Eine wahrscheinliche, noch kontrovers diskutierte Reduktion des „loop gain“ nach Apnoen bei älteren Menschen durch verringerte Sensitivität der $\mathrm{CO}_{2}$ - und $\mathrm{O}_{2}$-sensiblen Neurone im Glomus caroticum, bei Frauen auch infolge verringerten Atemantriebs durch reduziertes Progesteron, gleicht offensichtlich die strukturellen Veränderungen der oberen Atemwege nicht genügend aus, um die Anzahl repetitiver Apnoen nach Arousals wieder zu reduzieren [22].

Trotz der Bekanntheit dieser Phänomene und der altersphysiologisch eindeutigen Zunahme obstruktiver Apnoen mit dem Älterwerden gibt es noch keine neu definierten Grenzwerte für den AHI bei älteren Menschen, also z. B., dass ein AHI bis 15 bei Hochbetagten noch als normal angesehen werden könnte. Die Verantwortung, die klinische und damit therapiewürdige Bedeutung eines erhöhten Apnoe-Index bei Senioren zu beurteilen, bleibt daher beim behandelnden Arzt.

Dies gilt auch, wenn man zum Apnoe-Hypopnoe-Index als Marker noch 
den Sauerstoff-Entsättigungsindex hinzuzieht und insbesondere die durchschnittliche Sauerstoffsättigung im Blut $\left(\mathrm{SaO}_{2}\right)$ betrachtet. Aufgrund der geringeren Lungenkapazität im Alter durch Verlust elastischer Fasern und den Abbau am Sauerstoffaustausch teilnehmenden Lungengewebes kommt es altersphysiologisch $\mathrm{zu}$ einer Absenkung der durchschnittlichen $\mathrm{SaO}_{2}$ beim älteren Menschen. Eine Durchschnitts $\mathrm{SaO}_{2}$ von 93-92\% im Schlaf darf bei über 70-Jährigen in der Nacht als normal angesehen werden ([23]; • Abb. 1; • Tab. 2).

\section{Fazit}

Es gibt einige durch das Älterwerden bedingte physiologische Veränderungen des Schlafes und der Atmung im Schlaf, die bei Menschen im „best ager" (>55-70) - und im höheren Lebensalter zum Tragen kommen. Grundzüge dieser Veränderungen, wie im vorliegenden Artikel beschrieben, sollten jedem Mediziner und allen behandelnden Pflegekräften bekannt sein, damit Fehleinschätzungen, voreilig gestellte Diagnosen und daraus resultierende unnötige, vorwiegend medikamentöse Therapien reduziert werden können. Aufgabe aller aktiven Kräfte im Gesundheitswesen ist es, bei echten oder vermeintlichen Schlafstörungen das Gespräch mit dem älteren Patienten zu suchen und ihn quasi im physiologischen Alterungsprozess zu begleiten, bei deutlich pathologischen Veränderungen hingegen therapeutisch einzugreifen und nicht auf eine Laissez-faireSchiene zu geraten, die alle Störungen des Schlafes als altersbedingt abtut.

\section{Korrespondenzadresse}

Univ. Prof. Dr. med. Nikolaus Netzer, MD
PhD
Hermann Buhl Institut für Hypoxie und
Schlafmedizinforschung, Institut für
Sportwissenschaften, Fakultät für Psychologie
und Sportwissenschaften, Leopold Franzens
Universität Innsbruck
Bad Aibling, Deutschland
nikolaus.netzer@uibk.ac.at

Funding. Open access funding provided by University of Innsbruck and Medical University of Innsbruck.

\section{Einhaltung ethischer Richtlinien}

Interessenkonflikt. N. Netzer, S. Pramsohler und H. Frohnhofen geben an, dass kein Interessenkonflikt besteht.

Dieser Beitrag beinhaltet keine von den Autoren durchgeführten Studien an Menschen oder Tieren.

Open Access Dieser Artikel wird unter der Creative Commons Namensnennung 4.0 International Lizenz (http://creativecommons.org/licenses/by/4.0/deed. de) veröffentlicht, welche die Nutzung, Vervielfältigung, Bearbeitung, Verbreitung und Wiedergabe in jeglichem Medium und Format erlaubt, sofern Sie den/die ursprünglichen Autor(en) und die Quelle ordnungsgemäß nennen, einen Link zur Creative Commons Lizenz beifügen und angeben, ob Änderungen vorgenommen wurden.

\section{Literatur}

1. Normal Aging BDL (2011) Principles and Practice of sleep medicine. In: Kryger MH, Roth T, Dement WC (Hrsg) Philadelphia: Elsevier, 5. Aufl., S 27-31

2. Netzer NC (2014) Geriatric Sleep Medicine. In: Competencies in Sleep Medicine. York, Bd. 2014. Springer, Heidelberg/New York

3. Bliwise DL (1993) Sleep in normal aging and dementia. Sleep 16:40-81

4. Redline S, Kichner HL, Quan SF et al (2004) The effects of age, sex, ethnicity and sleep disordered breathing on sleep architecture. Arch Intern Med 164:406-418

5. Ohayon MM, Carscadon MA (2004) Guilleminault $c$, et al. Meta Analysis of quantitative sleep parameters from childhood to old age in healthy individuals: developing normative sleep values across human life span. Sleep 27:1255-1273

6. Floyd JA, Janisse JJ, Jenuwine ES et al (2007) Changes in REM sleep percentage over the adult life span. Sleep 30:829-836

7. Silber MH, Ancoli-Israel S, Bonnet MH et al (2007) The visual scoring of sleep in adults. J Clin Sleep Med 3:121-131

8. Marner L, Nyengaard JR, Tang Y, Pakkenberg B (2003) Marked loss of myelinated nerve fibers in the human brain with age. J Comp Neurol 21;462(2):144-152 (Jul)

9. Boselli M, Parrino L, Smieri A et al (1998) Effect of age on EEG arousal in normal sleep. Sleep 21:351-357

10. Klerman EB, Davis JB, Duffy JF et al (2004) Older people awaken more frequently but fall back asleep at the same rate as younger people. Sleep 27:793-798

11. Czeisler CA, Duffy JF, Shanahn TL et al (1999) Stability, precision and near 24-hour period of the human circadian pacemaker. Science 284:2177-2181

12. Koh K, Evans JM, Hendricks JC et al (2006) A drosophila model for age associated changes in sleep wake cycle. Proc Natl Acad Sci Usa 103:13843-13847

13. Dijk DJ, Duffy JF, Czeisler CA (2001) Age related increase in awakenings: impaired consolidation of nonREM sleep at all circadian phases. Sleep 30:1437-1443

14. Carrier J, Monk TH, Buysse DJ et al (1996) Including a 6 hour phase advance in the elderly: effects on sleep and temperature. J Sleep Res 5:99-105
15. Bliwise DL (1990) Sleep and circadian rhythm disorders in aging and dementia. In: Turek $F$, Zee $P$ (Hrsg) Regulation of sleep and circadian rhythms. Marcel Dekker, New York, S487-525

16. Zee P, Rosenberg RS, Turek FW (1992) Effects of aging on entrainment and rate or resynchronization of circadian locomotor activity. Am J Physiol 263:1099-1103

17. Nygard M, Hill RH, Wikstrom MA et al (2005) Age related changes in properties of the mouse suprachiasmaticus nucleus in vitro. Brain Res Bull 65:149-154

18. Hoban TF, Bliwise DL. Ontogeny. In Kushida C, editor. Obstructive sleep apnea: pathophysiology, comorbidities and consequences. New York: Informa Healthcare 2007. pp. 39-59

19. Eikerman M, Jordan AS, Chamberlin NL et al (2007) The influence of aging on pharyngeal collapsibility during sleep. Chest 131:1702-1709

20. Popovic RM, White DP (1985) Upper airway muscle activity in normal women: influence of hormonal status. J Appl Physiol 1998(Mar;84(3):1055-1062

21. Netzer NC, Eliasson AH, Strohl KP (2003) Women with sleep apnea have lower levels of sex hormones. Sleep Breath 7(1):25-29 (Mar)

22. Wellman A, Malhotra A, Jordan AS et al (2007) Chemical control stability in the elderly. J Physiol 581:291-298

23. Netzer N, Eliasson AH, Netzer C, Kristo DA (2001) Overnight pulse oximetry for sleep-disordered breathing in adults: a review. Chest 120(2):625-33. Review.

24. Netzer NC Chair, Ancoli-Israel S Co-Chair, Bliwise DL, Fulda S, Roffe C, Almeida F, Onen H, Onen F, Raschke F, Martinez Garcia MA, Frohnhofen $\mathrm{H}^{1}$ (2016) Principles of practice parameters for the treatment of sleep disordered breathing in the elderly and frail elderly: the consensus of the International Geriatric Sleep Medicine Task Force. Eur Respir J 48(4):992-1018. https://doi.org/10. 1183/13993003.01975-2015. Epub 2016 Jul 28. 